\title{
The Associated Factors of Anxiety and Depressive Symptoms in COVID-19 Patients Hospitalized in Wuhan, China
}

\author{
Xueyi $\mathrm{Li}^{1} \cdot$ Jun $\operatorname{Tian}^{1} \cdot$ Qun Xu${ }^{1}$ \\ Accepted: 15 November 2020/ Published online: 23 November 2020 \\ (C) Springer Science+Business Media, LLC, part of Springer Nature 2020
}

\begin{abstract}
This study was aimed to investigate the prevalence and factors associated with anxiety and depressive symptoms among hospitalized patients with COVID-19 during the epidemic outbreak in Wuhan, China. A total of 99 COVID-19 patients were recruited and completed the Hospital Anxiety and Depression Scale (HADS) and the modified Medical Research Council (mMRC) Scale. Results showed there was no significant difference in anxiety or depressive symptoms between male and female. Patients aged 46-60 years old had a higher ratio of both anxiety and depressive symptoms. Besides, patients whose hospital stays was longer than 14 days had a higher risk of depressive symptoms than those stays was less than 7 days. There was no significant difference in the correlation between level of dyspnea and the levels of anxiety or depressive symptoms. In conclusion, COVID-19 patients might have anxiety and depressive symptoms during hospitalization. Clinicians should pay attention to the middle age group and patients with longer hospital stays.
\end{abstract}

Keywords COVID-19 · Anxiety symptom · Depressive symptom · Dyspnea $\cdot$ Patient

\section{Introduction}

Since the outbreak of the coronavirus disease 2019 (COVID-19) in Wuhan, China, the rapid development of the epidemic has aroused the public concern worldwide. According to the World Health Organization [1] report, there had been 40,114,293 confirmed cases of COVID19 in 235 countries as of 20 , October, 2020. Those patients inevitably suffered from both physical symptoms and psychological pressure. The most common physical symptoms were fever (87.9\%), fatigue (69.6\%), dry cough (67.7\%), and myalgia (34.8\%) in China [2]. A

Qun Xu

xuqun2018@whu.edu.cn

1 Department of Rehabilitation Medicine, Zhongnan Hospital of Wuhan University, 169 Donghu Road, Wuchang District, Wuhan 430071 Hubei Province, People's Republic of China 
proportion of confirmed cases were characterized by dyspnea and hypoxemia. Similar to patients reported during the epidemic of severe acute respiratory syndrome (SARS) and the Middle East respiratory syndrome (MERS), COVID-19 patients were likely to develop the depressive and anxiety symptoms, as well as other psychiatric disorders during their quarantine and hospitalization [3].

For COVID-19 patients, several stressors were reported related to the psychiatric implications, such as prognosis of COVID-19 [4], isolation with a limited social support [5], limited information about the epidemic [5], financial burden [4], and stigma [5]. Furthermore, the stress of the overload healthcare staff [6], widespread fear, and public concern [7] may exacerbate patients' psychiatric symptoms (e.g., anxiety and depressive symptoms).

Dyspnea, a subjective syndrome of breathing discomfort that varies in intensity, is associated with interactions among multiple physiological, psychological, social, and environmental factors [8]. With an aggravation of breathing discomfort, patients may consider breathlessness as a threat associated with anxiety or depressive symptoms, which may induce secondary physical responses. Therefore, dyspnea may be associated with anxiety or depressive symptoms in COVID-19 patients during their hospitalization.

It is of great significance to investigate the psychiatric symptoms of confirmed COVID-19 patients during their hospitalization, to better understand the impact of SARS-CoV-2 in the acute stage. In the present study, we aimed to investigate the prevalence and factors associated with anxiety and depressive symptoms among hospitalized patients with COVID-19 in Wuhan, China.

\section{Methods}

\section{Study Design and Participants}

This cross-sectional study was conducted in Leishenshan Hospital, one of the new-built epidemic centers in Wuhan at the early stage of the outbreak. The inclusion criteria were as follows: (1) the nucleic acid test was positive; (2) patients with fever and respiratory symptoms; (3) computed tomography (CT) of chest was diagnosed of viral pneumonia; (4) being able to complete the modified Medical Research Council (mMRC) scale; and (5) patients who aged $\geq 18$ years old. The exclusion criteria were as follows: (1) respiratory rate $\geq 30$ breaths/ min; (2) oxygen saturation $\leq 93 \%$; (3) $\mathrm{PaO}_{2} / \mathrm{FiO}_{2} \leq 300 \mathrm{mmHg}$; (4) those with history of cognitive disorder, emotion disorder or chronic respiratory diseases. A total of 99 participants were enrolled in this survey from February 18 to March 18, 2020. Collected information included the demographic characteristics, history of COVID-19, chronic respiratory diseases, laboratory results, dyspnea measured by the mMRC scale score, and Hospital Anxiety and Depression Scale (HADS) score. This study was performed in accordance with the Declaration of Helsinki. Informed consent was obtained from all participants prior to commencing the study. Ethical approval of the study was obtained from the Ethics Committee of Zhongnan Hospital of Wuhan University (Wuhan, China).

\section{Measurement of Anxiety and Depressive Symptoms}

HADS is a self-rating scale comprised of two subscales. Each subscale includes seven intermingled items, and four ordinal choices are present in each item. According to this scoring 
system, participants with $0-7$ points in a subscale are defined as normal, with 8-10 points are classified as borderline abnormal, and with 11-21 scores are described as abnormal cases.

HADS has demonstrated satisfactory psychometric properties in different groups; in primary care patients, cognitively intact nursing home patients, cancer inpatients, and in general populations [9]. Additionally, descriptions in the HADS mainly centre on the recent feeling and do not mention any physical symptoms which may related to anxiety and depression, like dyspnea and fatigue. The setting has the advantage to focus on the psychometric problems and reduce the disturbance from somatic problems on the result. Studies concentrated on psychometric properties of HADS showed that it has satisfactory internal reliability [10] and adequate diagnostic accuracy [11].

\section{Measurement of Dyspnea}

In the present study, the severity of dyspnea was measured by the mMRC scale score $[12,13]$. The mMRC scale includes five grades $(0-4)$ of various physiological activities that provoke dyspnea. Details of this scoring system are as follows: 0 point, not troubled with breathlessness except with strenuous exercise; 1 point, troubled by shortness of breath when hurrying on the level or walking up a slight hill; 2 points, for walking more slowly than people of the same age on level surface because of breathlessness, or has to stop for breath when walking at own pace on level surface; 3 points, one had to stop for breath after walking about 100 yards or after a few minutes on level; and 4 points, for cases being extremely breathless to leave the room or breathless when dressing or undressing.

\section{Statistical Analysis}

The data were statistically analyzed using SPSS 20.0 software (IBM, Armonk, NY, USA). Counting data were expressed as frequency, and measured data were presented as mean \pm standard deviation. Chi-square test was applied to analyze the differences between levels of anxiety and depressive symptoms, as well as their risk factors. Then, multivariate logistic regression analysis was employed to calculate contribution of the statistically significant variables to the level of anxiety or depressive symptoms. The correlation between anxiety and depression subscales scores was analyzed by Spearman's Rank correlation analysis. $P<0.05$ was considered statistically significant.

\section{Results}

\section{Patients' Clinical Characteristics}

This study included a total of 99 qualified questionnaires, which were fulfilled by confirmed COVID-19 patients when they were in hospital. The patients' demographic, clinical, and laboratory characteristics are shown in Table 1. The patients' median age was 51.4 years old (range, 30-73 years old), and 54 of 99 (54.5\%) patients were male. The mean value of hospital stays until investigation was 12.4 days. It was found that $38(38.4 \%)$ patients had chronic respiratory diseases, and hypertension (26/38) was the most common disease. For the basic vital signs, the body temperature was $36.63 \pm 0.56{ }^{\circ} \mathrm{C}$, heart rate was $81.3 \pm 9.5 \mathrm{bpm}$, and respiratory rate was $19.80 \pm 3.01$ breaths $/ \mathrm{min}$. The first symptoms reported by COVID-19 
Table 1 Demographic, clinical, and laboratory characteristics of patients with COVID-19

\begin{tabular}{|c|c|c|}
\hline Characteristics & $N(\%)$ & Mean $\pm \mathrm{SD}$ \\
\hline Age & 99 & $51.4 \pm 11.9$ \\
\hline $30-45$ & $33(33.3 \%)$ & \\
\hline $46-60$ & $40(40.4 \%)$ & \\
\hline $60-73$ & $26(26.3 \%)$ & \\
\hline \multicolumn{3}{|l|}{ Gender } \\
\hline Female & $45(45.5 \%)$ & \\
\hline Male & $54(54.5 \%)$ & \\
\hline \multicolumn{3}{|l|}{ Time since onset of symptoms } \\
\hline $1-7$ days & $34(34.3 \%)$ & \\
\hline $8-14$ days & $26(26.3 \%)$ & \\
\hline$>14$ days & $39(39.4 \%)$ & \\
\hline Chronic respiratory diseases & $38(38.4 \%)$ & \\
\hline Hypertension & $26(26.3 \%)$ & \\
\hline Diabetes & $5(5.0 \%)$ & \\
\hline Hyperuricemia & $4(4.0 \%)$ & \\
\hline Cancer & $4(4.0 \%)$ & \\
\hline Hyperlipidemia & $2(2.0 \%)$ & \\
\hline COPD & $2(2.0 \%)$ & \\
\hline Hepatitis B & $1(1.0 \%)$ & \\
\hline \multicolumn{3}{|l|}{ Signs and symptoms at admission } \\
\hline Fever & $82(82.8 \%)$ & \\
\hline Fatigue & $49(49.4 \%)$ & \\
\hline Cough & $32(32.3 \%)$ & \\
\hline Asthma & $22(22.2 \%)$ & \\
\hline Chest distress & $8(8.1 \%)$ & \\
\hline No symptom & $4(4.0 \%)$ & \\
\hline Headache & $3(3.0 \%)$ & \\
\hline Anorexia & $2(2.0 \%)$ & \\
\hline Diarrhea & $2(2.0 \%)$ & \\
\hline Emesis & $1(1.0 \%)$ & \\
\hline Anhelation & $1(1.0 \%)$ & \\
\hline \multicolumn{3}{|l|}{ Laboratory results } \\
\hline White blood cell count $\left(* 10^{9} / \mathrm{L}\right)$ & 99 & $5.51 \pm 2.10$ \\
\hline Neutrophile granulocyte count $\left(* 10^{9} / \mathrm{L}\right)$ & 99 & $3.39 \pm 1.68$ \\
\hline Leukomonocyte count $\left(* 10^{9} / \mathrm{L}\right)$ & 99 & $1.54 \pm 0.73$ \\
\hline Mononuclear leucocyte count $\left(* 10^{9} / \mathrm{L}\right)$ & 98 & $0.53 \pm 0.19$ \\
\hline Thrombocyte count $\left(* 10^{9} / \mathrm{L}\right)$ & 96 & $235.8 \pm 85.9$ \\
\hline D-dimer $(\mathrm{ng} / \mathrm{ml})$ & 99 & $1484.7 \pm 3194.7$ \\
\hline C-reactive protein $(\mathrm{ng} / \mathrm{ml})$ & 96 & $6.16 \pm 12.59$ \\
\hline
\end{tabular}

$C O P D$ chronic obstructive pulmonary disease

patients were as follows: fever (82/99), fatigue (49/99), cough (32/99), asthma (22/99), chest distress (8/99), no symptom (4/99), headache (3/99), anorexia (2/99), diarrhea (2/99), emesis (1/99), and anhelation (1/99).

\section{Levels of Anxiety Symptoms, Depressive Symptoms and Dyspnea}

The data related to different levels of dyspnea, anxiety and depressive symptoms in patients with COVID-19 are presented in Table 2 . The mean value of anxiety score was 6.69 points, and depression score was 8.27 points. For anxiety symptoms, $26(26.3 \%)$ patients were borderline cases, and $15(15.2 \%)$ patients were evaluated as abnormal. For depressive 
Table 2 Levels of dyspnea, anxiety and depressive symptoms

\begin{tabular}{llr}
\hline Scale & $N$ & Rate $(\%)$ \\
\hline Anxiety score & & 58.6 \\
$0-7$ & 58 & 26.3 \\
$8-10$ & 26 & 15.2 \\
$>10$ & 15 & 49.5 \\
Depression score & & 21.2 \\
$0-7$ & 49 & 29.3 \\
$8-10$ & 21 & 27.3 \\
$>10$ & 29 & 31.3 \\
Dyspnea scale & & 18.2 \\
0 & 27 & 16.2 \\
1 & 31 & 7.1 \\
2 & 18 & \\
4
\end{tabular}

symptoms, $21(21.2 \%)$ patients were borderline cases, and 29 (29.3\%) patients were classified as abnormal. For dyspnea, $27(27.3 \%)$ patients did not feel any difficulty in breathing, and 31 $(31.3 \%)$ patients were troubled by shortness of breath during walking. In addition, 18 (18.2\%), $16(16.2 \%)$, and $7(7.1 \%)$ patients achieved 2, 3, and 4 points, respectively.

\section{Level of Anxiety Symptoms and Risk Factors}

For anxiety symptoms, no significant differences were observed in gender $\left(\chi^{2}=3.197, p=\right.$ $0.074)$ and hospital stays $\left(\chi^{2}=1.317, p=0.536\right)$. Results showed that patients who aged 4660 years old had a higher rate of anxiety symptoms than those who aged above 60 years old $\left(\chi^{2}=7.580, p=0.006\right)$. As we set $p<0.1$ as the inclusion criteria into the logistic regression model, gender and age were included in the anxiety-symptom-associated multivariate logistic regression model. Additionally, the results showed that the risk of anxiety symptoms in patients who aged 46-60 years old was 3.83 times (95\% confidence interval $(C I), 1.23-$ 11.88) higher than those who aged above 60 years old (Table 3 ).

\section{Level of Depressive Symptoms and Risk Factors}

For depressive symptoms, 21 (21.2\%) patients were classified as borderline cases, and 29 (29.3\%) patients were abnormal (Table 2). Thus, $50(50.01 \%)$ patients were assessed as having depressive symptoms. There was no significant difference between male and female patients in depressive symptoms $\left(\chi^{2}=0.264, p=0.688\right)$. Significant differences were found in age $\left(\chi^{2}=6.311, p=0.044\right)$ and hospital stays $\left(\chi^{2}=8.559, p=0.014\right)$. In contrast to the results of anxiety symptoms, the Chi-square test showed that patients who aged 46-60 years old had a higher rate of depressive symptoms than those who aged 30 45 years old $\left(\chi^{2}=6.154, p=0.013\right)$. For hospital stays until investigation, compared with patients whose hospital stays were $\leq 7$ days, patients staying more than 14 days had a significantly higher rate of depressive symptoms $\left(\chi^{2}=8.557, p=0.003\right)$. There was no interaction effect between age and hospital stays in depressive symptoms $(F=0.134, p=$ 0.969). The multivariate logistic regression model showed the risk of depressive 
Table 3 Anxiety-symptom-associated factors in COVID-19 patients

\begin{tabular}{|c|c|c|c|c|c|}
\hline \multirow[t]{2}{*}{ Factors } & \multirow[t]{2}{*}{ Non-anxiety } & \multirow[t]{2}{*}{ Anxiety } & \multirow[t]{2}{*}{$p$} & \multicolumn{2}{|c|}{ Multivariate logistic regression analysis } \\
\hline & & & & OR $(95 \% C I)$ & $p$ \\
\hline Gender & & & 0.074 & & \\
\hline Female & $22(48.9 \%)$ & $23(51.1 \%)$ & & $1.96(0.80-4.78)$ & 0.14 \\
\hline Male & $36(66.7 \%)$ & $18(33.3 \%)$ & & 1.0 & - \\
\hline Age (years old) & & & 0.018 & & \\
\hline $30-45$ & $21(63.6 \%)$ & $12(36.4 \%)$ & & $1.42(0.41-4.85)$ & 0.578 \\
\hline $46-60$ & $17(42.5 \%)$ & $23(57.5 \%)$ & & $3.83(1.23-11.88)$ & 0.02 \\
\hline$>60$ & $20(76.9 \%)$ & $6(23.1 \%)$ & & 1.0 & - \\
\hline Hospital stays until investigation & & & 0.536 & & \\
\hline$\leq 7$ & $22(64.7 \%)$ & $12(35.3 \%)$ & & & \\
\hline $8-14$ & $13(50.0 \%)$ & $13(50.0 \%)$ & & & \\
\hline$>14$ & $23(59.0 \%)$ & $16(41.0 \%)$ & & & \\
\hline Dyspnea scale & & & 0.751 & & \\
\hline 0 & $14(58.3 \%)$ & $10(41.7 \%)$ & & & \\
\hline 1 & $17(53.1 \%)$ & $15(46.9 \%)$ & & & \\
\hline 2 & $13(68.4 \%)$ & $6(31.6 \%)$ & & & \\
\hline $3-4$ & $14(58.3 \%)$ & $10(41.7 \%)$ & & & \\
\hline
\end{tabular}

symptoms in patients who stayed $>14$ days, was 4.89 times $(95 \%$ CI, 1.72-13.94) higher than those stayed $\leq 7$ days in hospital (Table 4 ).

\section{Level of Dyspnea and its Correlation with Anxiety or Depressive Symptoms}

The level of dyspnea is summarized in Table 2. The Spearman's Rank correlation analysis showed that the level of dyspnea was not correlated with the anxiety scores in COVID-19 patients $\left(p=0.083, \mathrm{r}_{\mathrm{s}}=0.175\right)$. For depressive symptoms, there was no significant correlation between levels of dyspnea and depression scores $(p=0.184)$.

Table 4 Depressive-symptom-associated factors in COVID-19 patients

\begin{tabular}{|c|c|c|c|c|c|}
\hline \multirow[t]{2}{*}{ Factors } & \multirow[t]{2}{*}{ Normal } & \multirow[t]{2}{*}{ Depressive } & \multirow[t]{2}{*}{$p$} & \multicolumn{2}{|c|}{ Multiple logistic regression analysis } \\
\hline & & & & OR $(95 \% C I)$ & $p$ \\
\hline Gender & & & 0.688 & & \\
\hline Male & $28(51.9 \%)$ & $26(48.1 \%)$ & & & \\
\hline Female & $21(46.7 \%)$ & $24(53.3 \%)$ & & & \\
\hline Age (years old) & & & 0.044 & & \\
\hline $30-45$ & $22(66.7 \%)$ & $11(33.3 \%)$ & & $0.49(0.16-1.50)$ & 0.211 \\
\hline $46-60$ & $15(37.5 \%)$ & $25(62.5 \%)$ & & $1.94(0.65-5.77)$ & 0.232 \\
\hline$>60$ & $12(46.2 \%)$ & $14(53.8 \%)$ & & 1.0 & - \\
\hline Time since admission & & & 0.014 & & \\
\hline$\leq 7$ & $23(67.6 \%)$ & $11(32.4 \%)$ & & 1.0 & - \\
\hline $8-14$ & $13(50.0 \%$ & $13(50.0 \%)$ & & $2.19(0.73-6.57)$ & 0.163 \\
\hline$>14$ & $13(33.3 \%)$ & $26(66.7 \%)$ & & $4.89(1.72-13.94)$ & 0.003 \\
\hline Dyspnea scale & & & 0.768 & & \\
\hline 0 & $12(50.0 \%)$ & $12(50.0 \%)$ & & & \\
\hline 1 & $14(43.8 \%)$ & $18(56.3 \%)$ & & & \\
\hline 2 & $9(47.4 \%)$ & $10(52.6 \%)$ & & & \\
\hline $3-4$ & $14(58.3 \%)$ & $10(41.7 \%)$ & & & \\
\hline
\end{tabular}




\section{Discussion}

This cross-sectional study showed that the mean score of anxiety symptoms in COVID-19 patients was $6.69 \pm 5.01$ points during their hospitalization, in which $26.3 \%$ and $15.2 \%$ of cases were borderline and abnormal. The depression score was $8.27 \pm 5.35$ points, and the portion of borderline and abnormal cases was $21.2 \%$ and $29.3 \%$, respectively. The abovementioned results indicated that COVID-19 infection may result in anxiety and depressive symptoms in such patients during their hospitalization. The outcomes of the current research were consistent with psychiatric findings of SARS and MERS patients when they were in the acute stage [3]. To our knowledge, few studies have concentrated on COVID-19 patients' psychological status. In the present study, we reported a high prevalence of anxiety and depressive symptoms in COVID-19 hospitalized patients. A similar study reported that $34.72 \%$ of COVID-19 patients had anxiety symptoms and $28.47 \%$ of patients had depressive symptoms [14], which were consistent with our findings.

Additionally, a significantly positive correlation was found between anxiety and depression scores of HADS. This indicated that COVID-19 patients with the symptoms of anxiety were prone to companion with depressive symptoms, and vice versa.

We found that the anxiety-symptom-associated factors were age and gender, which was consistent with Wang et al.'s study [15]. In their study, age was taken as an anxiety-associated factor into account in healthy individuals during the COVID-19 outbreak. Our study showed that the rate of anxiety symptoms was higher in patients who aged 46-60 years old. In China, individuals aging 46-60 years old often have the main responsibility and act as backbone of their family. Despite the common COVID-19-related symptoms, they may have concern about a temporary disruption to the economy. Moreover, females have probably a higher risk of anxiety than males. Acting as a leading caregiver in family and being more sensitive to separation [15], women are more prone to have anxiety symptoms.

Dyspnea may affect emotions and increase psychological stress. Numerous studies concentrated on patients with chronic obstructive pulmonary disease (COPD) have reported a positive correlation between anxiety and dyspnea [16-18]. Anxiety can trigger hyperventilation, further provoking anxiety. Limited by the small sample size, our study found that the level of dyspnea has no correlation with the level of anxiety symptoms, while a tendency was obvious. In patients with COVID-19, dyspnea is one of the prevalent symptoms at the onset of disease, and dyspnea increases as pneumonia deteriorates [19]. Further large sample size studies are required to confirm the positive association between dyspnea and anxiety symptoms in COVID-19 patients.

We found that age and hospital stays are factors affecting the rate of depressive symptoms in COVID-19 patients. As same as anxiety symptoms, patients who aged 46-60 years old also had a higher rate of depressive symptoms. As our results unveiled that depressive symptoms was associated with anxiety symptoms, and the stressors that influenced anxiety symptoms could increase the possibility of depressive symptoms, including financial burden and family responsibilities. The present study demonstrated that time since admission or length of hospitalization could be associated with depressive symptoms, which was consist with Brooks et al.'s study [5] and Papaioannou et al.'s research [20]. Papaioannou et al. [20] reported that for COPD patients, the length of hospitalization was correlated with depression scores. It is noteworthy that hospitalization during COVID-19 outbreak is quite different from the common experience for the public. It may be their first time to be quarantined in a hospital. The above-mentioned factors could easily exacerbate the depressive symptoms, especially when length of hospitalization was longer. 
The current study did not support a correlation between dyspnea and depressive symptoms in COVID-19 patients, while a number of previous studies showed a significant association between depressive symptoms and dyspnea stage in COPD patients [20, 21]. COPD is a chronic respiratory disease caused inactivity and function impairment, which may lead to selfdoubt and depression [20]. Although COVID-19 is an acute respiratory disease with high infectivity, difference in disease progression may be a reason justifying discrepancy between outcomes related to dyspnea and depressive symptoms. It is therefore essential to conduct a study with larger sample size to explore relationship between dyspnea and depressive symptoms in COVID-19 patients.

\section{Limitations}

The present study had several limitations. First, small number of participants might limit the results, especially the correlation between anxiety symptoms and dyspnea. Second, all participants in our study were recruited from one hospital and one city, which might cause bias. Third, we only evaluated COVID-19 patients' psychological status when they were hospitalized. Follow-up of anxiety and depressive symptoms needs to be conducted in the future study.

\section{Conclusions}

A proportion of COVID-19 patients might have anxiety and depressive symptoms during hospitalization. Age and hospital stays are risk factors associating with anxiety or depressive symptoms. Hence, it is highly vital to care about COVID-19 patients' mental health when they were hospitalized.

Authors' Contribution Qun Xu designed the study, wrote the protocol, and conducted the survey. Xueyi Li performed statistical analysis, and wrote the first draft of the manuscript. All the authors read and approved the submitted version of the manuscript.

\section{Compliance with Ethical Standards}

Conflict of Interest The authors declare that there is no conflict of interest.

Ethics Statement This cross-sectional study was performed in accordance with the Declaration of Helsinki. Informed consent was obtained from all participants prior to commencing the study. Ethical approval of the study was obtained from the Ethics Committee of Zhongnan Hospital of Wuhan University (Wuhan, China).

Code Availability Not applicable.

\section{References}

1. World Health Organization. Coronavirus disease (COVID-19) pandemic. WHO. https://www.who.int/ emergencies/diseases/novel-coronavirus-2019. Accessed 29 May 2020.

2. Han Q, Lin Q, Jin S, You L. Coronavirus 2019-nCoV: a brief perspective from the front line. J Infect. 2020;80:373-7. 
3. Rogers JP, Chesney E, Oliver D, Pollak TA, McGuire P, Fusar-Poli P, et al. Psychiatric and neuropsychiatric presentations associated with severe coronavirus infections: a systematic review and meta-analysis with comparison to the COVID-19 pandemic. Lancet Psychiatry. 2020;7:611-27.

4. Xiang YT, Yang Y, Li W, Zhang L, Zhang Q, Cheung T, et al. Timely mental health care for the 2019 novel coronavirus outbreak is urgently needed. Lancet Psychiatry. 2020;7:228-9.

5. Brooks SK, Webster RK, Smith LE, Woodland L, Wessely S, Greenberg N, et al. The psychological impact of quarantine and how to reduce it: rapid review of the evidence. Lancet. 2020;395:912-20.

6. Greenberg N, Docherty M, Gnanapragasam S, Wessely S. Managing mental health challenges faced by healthcare workers during covid-19 pandemic. Bmj. 2020;368:m1211.

7. Asmundson GJG, Taylor S. Coronaphobia: fear and the 2019-nCoV outbreak. J Anxiety Disord. 2020;70: 102196.

8. Parshall MB, Schwartzstein RM, Adams L, Banzett RB, Manning HL, Bourbeau J, et al. An official American Thoracic Society statement: update on the mechanisms, assessment, and management of dyspnea. Am J Respir Crit Care Med. 2012;185:435-52.

9. Bjelland I, Dahl AA, Haug TT, Neckelmann D. The validity of the hospital anxiety and depression scale. An updated literature review. J Psychosom Res. 2002;52:69-77.

10. Yang Z, Huang X, Liu X, Hou J, Wu W, Song A, et al. Psychometric properties and factor structure of the Chinese version of the hospital anxiety and depression scale in people living with HIV. Front Psych. 2019;10:346.

11. Norton S, Cosco T, Doyle F, Done J, Sacker A. The hospital anxiety and depression scale: a meta confirmatory factor analysis. J Psychosom Res. 2013;74:74-81.

12. Altose MD. Assessment and management of breathlessness. Chest. 1985;88:77s-83s.

13. Paz-Diaz H, Montes de Oca M, Lopez JM, Celli BR. Pulmonary rehabilitation improves depression, anxiety, dyspnea and health status in patients with COPD. Am J Phys Med Rehabil. 2007;86:30-6.

14. Kong X, Zheng, K, Tang M, Kong F, Zhou J, Diao L, et al. Prevalence and factors associated with depression and anxiety of hospitalized patients with COVID-19. medRxiv. 2020; 2020.2003.2024.20043075.

15. Wang Y, Di Y, Ye J, Wei W. Study on the public psychological states and its related factors during the outbreak of coronavirus disease 2019 (COVID-19) in some regions of China. Psychol Health Med. 2020:110 .

16. Gudmundsson G, Gislason T, Janson C, Lindberg E, Hallin R, Ulrik CS, et al. Risk factors for rehospitalisation in COPD: role of health status, anxiety and depression. Eur Respir J. 2005;26:414-9.

17. Yohannes AM, Baldwin RC, Connolly MJ. Depression and anxiety in elderly outpatients with chronic obstructive pulmonary disease: prevalence, and validation of the BASDEC screening questionnaire. Int J Geriatr Psychiatry. 2000;15:1090-6.

18. Yohannes AM, Junkes-Cunha M, Smith J, Vestbo J. Management of Dyspnea and Anxiety in chronic obstructive pulmonary disease: a critical review. J Am Med Dir Assoc. 2017; 18:1096.e1091-17.

19. Chen T, Wu D, Chen H, Yan W, Yang D, Chen G, et al. Clinical characteristics of 113 deceased patients with coronavirus disease 2019: retrospective study. Bmj. 2020;368:m1091.

20. Papaioannou AI, Bartziokas K, Tsikrika S, Karakontaki F, Kastanakis E, Banya W, et al. The impact of depressive symptoms on recovery and outcome of hospitalised COPD exacerbations. Eur Respir J. 2013;41: 815-23.

21. Schuler M, Wittmann M, Faller H, Schultz K. The interrelations among aspects of dyspnea and symptoms of depression in COPD patients - a network analysis. J Affect Disord. 2018;240:33-40.

Publisher's Note Springer Nature remains neutral with regard to jurisdictional claims in published maps and institutional affiliations.

Xueyi Li , a postgraduate student from Wuhan University, mainly does the research on rehabilitation after stroke. E-mail: 2018203030083@whu.edu.cn.

Jun Tian, an associate chief physician in the department of rehabilitation medicine, Wuhan University of Zhongnan Hospital, mainly does the research on integrated rehabilitation treatment on osteoarthrosis. E-mail: tianjun@znhospital.cn.

Qun xu is a $\mathrm{PhD}$ graduate from Huazhong University of Science and Technology and a physician in the department of rehabilitation medicine, Wuhan University of Zhongnan Hospital. His main research focuses on spasm after stroke. E-mail: xuqun2018@whu.edu.cn. 